\title{
Domaine de spécialisation, expérience professionnelle et stratégie de régulation cognitive chez des médecins des centres hospitaliers universitaires (CHU) de Côte-d'Ivoire
}

\author{
Kouame Ahou Aline Eveline, \\ Doctorante en Psychologie Génétique Différentielle, \\ Université Félix Houphouet Boigny, Abidjan-Cocody, Côte d'Ivoire
}

\section{Résumé}

Cette étude a été réalisée auprès de quarante trois (43) médecins de trois Centres Hospitaliers Universitaires (CHU) de Côte-d'Ivoire dont vingttrois (23) généralistes et vingt (20) spécialistes (12 pédiatres et 08 endocrinologues). L'objectif fut la mise en évidence de la relation entre le domaine de spécialisation, l'expérience professionnelle et la stratégie de régulation cognitive chez ces médecins. Par le biais de l'échelle de régulation cognitive conçu spécialement pour cette étude, nous avons réparti les médecins en fonction du type de stratégie de régulation mise en œuvre lors de l'activité de soignant. Un questionnaire et des entretiens avec les chefs de service ont permis de distinguer les différentes spécialisations et de relever les années de présence dans le service. Globalement, les résultats obtenus montrent que le domaine de spécialisation et l'expérience professionnelle influencent la mise en œuvre des stratégies de régulation. Les généralistes débutants mettent en œuvre une stratégie de régulation réactive tandis que les spécialistes expérimentés sont favorables à une stratégie de régulation proactive. Concernant l'expérience, les résultats indiquent qu'il y a une différence significative entre les débutants et les expérimentés. Toutefois, le présent travail n'a pu démontrer le lien entre le domaine de spécialisation et la stratégie de régulation cognitive. Les résultats obtenus ressortent la thèse selon laquelle le type de stratégie cognitive mis en œuvre lors des activités dépend des facteurs personnels, situationnels et environnementales.

Mots-clés: Métacognition, Autorégulation, Stratégie de Régulation, Spécialité Médicale, Expérience Professionnelle 


\title{
Field of Specialization, Professional Experience and Strategy of Cognitive Regulation in Doctors of University Hospitals Centers (CHU) of Ivory Coast
}

\author{
Kouame Ahou Aline Eveline, \\ Doctorante en Psychologie Génétique Différentielle, \\ Université Félix Houphouet Boigny, Abidjan-Cocody, Côte d'Ivoire
}

\begin{abstract}
This study was conducted with forty-three (43) physicians from three University Hospital of Côte d'Ivoire including twenty-three (23) general practitioners and twenty (20) specialists (12 pediatricians and 08 endocrinologists). The objective was to highlight the relationship between the field of specialization, professional experience and the regulatory strategy of physicians in the. Using the Cognitive Regulation Scale developed specifically for this study, we divided physicians according to the type of regulation strategy implemented during of the caring activity. A questionnaire and interviews with the heads of service made it possible to determine their different specializations and professional experiences. Overall, the results show that the area of specialization and professional experience influence the implementation of regulatory strategies. A significant difference between junior general practitioners and experienced specialists is revealed. The former are implementing a reactive regulation strategy while the latter are in favor of a proactive regulatory strategy. Regarding experience, the results indicate that there is a significant difference between beginners and experienced. However, the present work could not demonstrate the link between the field of specialization and the cognitive regulation strategy. The results obtained point to the thesis according to wich the type of cognitive strategies implementation during the activities depends on personal, situational and environmental factors.
\end{abstract}

Keywords: Metacognition, Self-regulation, Regulatory Strategy, Medical Specialty, Professional Experience 


\section{Introduction et Problematique}

L'homme a probablement toujours porté une réflexion sur son activité pour tenter de l'optimiser. Cette réflexion sur l'activité dans le but de l'optimiser s'est développée en science avec la création de l'ergonomie. Etant un être vivant en société, il est alors amené à agir lorsqu'il est confronté à diverses situations. Selon Gilbert Boss (2000), il y a toute une réflexion de caractère théorique, destinée à préparer la pensée qui orientera l'action de celui-ci. Pour comprendre ce phénomène, les travaux des psychologues cognitivistes tels que Swinnen, Heuer, Massion \& Casaer (2013) ont conduit à caractériser au moins deux types de contraintes (structurale ou fonctionnelle) qui pèsent sur le système cognitif. La première représente les différents composants et processus mis en œuvre par le système cognitif, et la seconde représente les caractéristiques et les représentations de chaque processus.

Ainsi, la cognition qui permet la prise de conscience par l'individu de son propre fonctionnement intellectuel fait appel à la métacognition. Cette dernière consiste à savoir relier de nouvelles connaissances à des savoirs antérieurs et à être capable de sélectionner des stratégies cognitives. Le concept de métacognition a donné lieu à deux branches d'étude distinctes, développementale et cognitive, avec des perspectives théoriques et des paradigmes expérimentaux différents (Son \& Schwartz, 2002 ; Schneider \& Lockl, 2002 ; Koriat, 2000).

Pour Son et Schwartz (op. cit), la perspective cognitive se distingue de la perspective développementale (orientée sur des processus développementaux) parce qu'elle s'intéresse à des processus déjà développés. Elle renvoie alors à la régulation de la cognition qui est la capacité de contrôle et d'ajustement de ses processus cognitifs ou processus d'apprentissage lors de l'exécution d'une tâche (Gombert (1990); Royer, Cisero \& Carlo (1993); Noël, Romainville \& Wolfs (1995)). C'est cette perspective cognitive de la métacognition qui constituera le sujet de notre propos dans cet article. Elles se réfèrent donc à l'utilisation délibérée de stratégies dans la visée d'ajuster sa cognition et prendre des décisions adaptées (Efklides, 2006 ; 2008).

Selon Morin (2000), la stratégie qui se trouve être au cœur des stratégies d'autorégulation cognitive peut se référer à la méthode d'action propre à un sujet en situation de résolution d'un problème. Elle se rapporte à la règle ou à la procédure permettant d'opérer une sélection par les options qui sont déjà disponibles, ou d'en construire une nouvelle. De ce fait, la stratégie qui renvoie à l'ensemble des manœuvres susceptibles de conduire à une adaptation réussie repose sur une démarche d'anticipation en vue d'atteindre un objectif. Elle nécessite compétence et initiative car elle suppose une certaine aptitude à entreprendre une action. Elle vise à choisir des actions, à les mettre en œuvre et à les coordonner afin d'obtenir un résultat. On s'imagine alors que, dès le début de l'action, la stratégie se prépare, afin de détecter 
l'élément nouveau ou l'inattendu et, ensuite, l'intégrer pour modifier ou enrichir son action. De ce point de vue, les activités qui nécessitent l'utilisation ou la mise en place d'une stratégie sont nombreuses car elles visent l'adaptation.

Nous souhaitons aborder dans notre travail les activités d'autorégulation menées par les individus dans le cadre de la réalisation de tâches spécifiques. Les recherches entreprises dans ce cadre visent à montrer que la stratégie d'autorégulation cognitive a trois principales composantes que sont les buts cognitifs, les processus métacognitifs et les processus comportementaux encore appelés stratégies de régulation (Zimmerman, 1986 ; 1989). Selon ces auteurs, la mise en œuvre de processus d'autorégulation cognitive apparait comme une variable décisive permettant, d'augmenter la performance au travail, de préserver le bien-être et de réussir dans l'apprentissage. Cependant, Boekaerts (1997) précise que la stratégie d'autorégulation cognitive repose en grande partie sur la stratégie de régulation et que cette dernière est au cœur de l'adaptation réussie et du succès. Autrement dit, cet auteur révèle que la troisième composante (la stratégie de régulation) de l'autorégulation cognitive est celle qui a le plus d'impact lorsqu'il s'agit de but à atteindre en terme de comportement à manifester. Ainsi, l'individu qui est capable de stratégie de régulation peut sélectionner les procédures les plus efficaces dans la perspective d'atteindre l'objectif déterminé, les contrôler et les réajuster si nécessaire.

Parler alors de stratégie de régulation au travail consiste à faire allusion à la performance à laquelle elle renvoie de façon pratique. En effet, les stratégies de régulations se basent sur la métacognition et impliquent des processus dirigés vers des buts, nécessitant d'utiliser, de traiter et de gérer l'information disponible dans l'environnement de façon stratégique, en fonction des buts à atteindre. Selon une conception longtemps prédominante du fonctionnement de l'esprit, les individus sont dotés d'un ensemble de capacités générales de raisonnement qui interviennent dans toutes les tâches cognitives quel qu'en soit le contenu spécifique. Ces tâches conduiront à des actions qui sont conçues en psychologie comme le déroulement d'un geste dont il est possible d'identifier trois étapes que sont la planification, la programmation et l'exécution (Houdé, Kayser, Koenig, Proust, Rastier, \& all. 2003). Seule la dernière étape est directement observable et introduit une modification dans l'environnement à travers des anticipations. Les deux premières, mentalement élaborées et antérieures au déclenchement de l'action, déterminent le but et la stratégie à adopter (planification) de même que le déroulement (programmation).

Brick, MacIntyre \& Campbell (2015) ayant travaillés sur la perspective cognitive de l'autorégulation chez des athlètes ont pu montrer que l'utilisation de stratégie de régulation permet aux athlètes d'atteindre un niveau optimal de 
performances. Les travaux de Locke \& Latham $(2002$; 2006) ont quant à eux révélé qu'un calibrage des buts influence la conduite des processus d'autorégulation puisque cette conduite est dirigée par un but. Les buts ainsi calibrés permettent de canaliser l'attention vers des activités pertinentes pour atteindre les buts fixés et, de ce fait, aident l'apprenant à distinguer les conduites prioritaires des conduites parasites sources de distraction. Ils favorisent également l'adoption d'une conduite stratégique afin de mobiliser le savoir pertinent ou de découvrir et d'intégrer le savoir requis pour parvenir à un apprentissage réussi. Ainsi, des éléments peuvent bien influencer l'utilisation de stratégies de régulation et orienter les actions de l'individu dans l'optique d'atteindre le but visé. De par leurs travaux, Buchs, Darnon, Quiamzade, Mugny \& Butera (2008) affirment que l'expression des désaccords conduit à les réguler. Cette régulation tient compte selon eux du contenu et de la compétence et fait donc apparaitre deux modes de régulation. Une régulation centrée sur la compréhension du problème et une régulation compétitive dont le but est défensif, comme une réponse sans plus.

Quant à Chalus (2000), il révèle que les individus développent le plus souvent une régulation proactive qui consiste en l'élaboration de procédure (activité de construction et de production) en vue d'anticiper des actions pour parvenir à l'efficacité, au détriment d'une régulation réactive (activité de production). La stratégie proactive est à l'origine des anticipations, elle peut être considérée comme une attitude ou un état d'esprit tourné vers le futur. La stratégie réactive consiste, sans anticipation, à répondre à une nouvelle contrainte en s'y soumettant comme à une injonction et partant sans créativité suffisante en retour. Selon Mahé de Boislandelle (1998), la proactivité se différencie de la réactivité par des changements de comportement qui traduisent des adaptations anticipatrices. C'est donc la stratégie proactive qui est très souvent associée au succès et à la réussite dus aux régulations cognitives que les auteurs ont soulignées. Au niveau professionnel, plusieurs emplois nécessitent des activités de production et de construction comme le stipule Samurcay \& Rabardel (2004). Toutefois, le comportement peut dépendre de feedbacks ou de but à atteindre. Cela ne change pas le fait qu'il renvoie à une seule et même réalité, celle de résoudre un problème bien précis. Cependant, il serait plus profitable d'orienter son action en se fixant des buts à atteindre plutôt que de fonctionner par essai erreur (Lemaire, 1999). Cela est d'autant plus nécessaire pour le médecin dont le domaine d'action est l'être humain car une erreur dans sa pratique peut être fatale pour ce dernier.

Le domaine de la médecine étant vaste, celle-ci est composée de plusieurs spécialités dans lesquels les différents médecins rencontrent plusieurs patients, et sont confrontés à diverses pathologies. Ils doivent de ce fait, être en mesure de développer une régulation proactive qui vise comme but la découverte de l'origine du mal du patient afin de lui proposer un 
traitement efficace. Adeyoyin, \& al. (2015) révèlent que la spécialisation professionnelle conduit à une meilleure satisfaction des employés et donc à une efficacité dans la tâche. La satisfaction de l'employé favorise alors un investissement plus accru et une meilleure performance. Cela pouvant être le fait d'une régulation plus adaptée. Duggan \& Payne (2008) stipulent qu'une meilleure connaissance du domaine favorise l'utilisation de stratégie qui permet une rapidité dans la prise de décision et une meilleure performance en ce qui concerne la tâche à accomplir. Cependant, il faut préciser que, bien que la spécialisation soit source de compétence, elle ne peut à elle seule orienter l'action et inspirer la démarche comportementale. Il faut donc une certaine connaissance des tâches à accomplir et de leur aboutissement. En effet, lorsque l'on débute dans une profession, on n'est sûr ni de notre action ni de notre manière de gérer les situations qui se présentent à nous. Après quelques années d'exercice, certains travailleurs apprennent à anticiper les difficultés. Cette anticipation est fonction de l'expérience professionnelle acquise au travail. Elle peut être assimilée au temps passé dans l'entreprise notamment à l'ancienneté qui naturellement produit du capital humain (Chiswick, 2003, Lemelin, 1998).

Des études empiriques confirment l'évidence que le capital humain des médecins a un effet sur la rapidité des soins. Olive (2011) révèle l'impact de l'ancienneté sur le succès en prouvant qu'après quelques années de services, les interprètes acquièrent de la confiance, apprennent à anticiper et à relativiser. Selon Gasparovic (2009), l'expérience professionnelle se mesure par l'ancienneté sur le marché du travail et révèle que celle des débutants est strictement inférieure à 3 ans. Les médecins expérimentés seront donc plus efficaces et obtiendront de bons résultats dans leur pratique quotidienne du fait de la répétition des cas qui ont souvent les mêmes causes et suivent la même évolution. Richard (1990) et Boekaerts (1997) s'accordant sur le fait que la régulation prépare au succès dans la réalisation d'une tâche et vise un but à atteindre. Il est donc nécessaire de situer la spécialisation et l'expérience dans le processus de mise en œuvre de cette régulation. De ce fait, la stratégie de régulation chez les médecins doit conduire à atteindre le but principal de cette corporation qui est de trouver l'origine du mal du patient et de lui proposer un traitement qui viendra à bout de son mal.

Ainsi, la capacité du médecin à prendre des initiatives, à planifier ses actions, à anticiper ses interventions afin d'obtenir la guérison de son patient dépend de sa connaissance du cas et de ses acquis du fait de ses actions antérieures. Plus ses acquis sont précis et spécifiques, plus il est à même d'entreprendre une régulation proactive dans l'accomplissement de ses tâches quotidienne. De plus, un médecin dont la formation est en adéquation avec son domaine d'action se sent efficace et apte à gérer toute situation qui se présente 
à lui aussi imprévisible qu'elle soit. Ce qui sera pour lui porteur d'expérience avec le temps.

Bien que souvent sollicités, les besoins de santé ayant changé, les établissements sanitaires ne sont aujourd'hui plus équipés pour parer à tous les cas de figure. De ce fait, les médecins sont confrontés à l'utilisation de stratégies cognitives en vue d'une adaptation. Cela consiste en la bonne gestion des connaissances pour une application réelle dans les différentes situations professionnelles. Le niveau actuel des principaux indicateurs de morbidité et de mortalité rend compte de l'état de santé général de la population, et donc de l'efficacité des stratégies utilisées par les médecins au quotidien. Il traduit dans une certaine mesure le niveau de la demande de soins de la population notamment, pour les couches les plus vulnérables (Bini, \& al. 2008).

Les médecins doivent donc agir selon le serment d'Hippocrate c'est-àdire sans tenir compte de l'origine sociale, ethnique et religieuse du patient. Néanmoins, ils sont confrontés à plusieurs difficultés dans leur pratique qui influencent leur prise de décision entre autre la soumission aux règlements et procédures limitant les prises d'initiative, de même que le manque de matériel. De façon pratique, cette étude va nous renseigner sur le mode de régulation préférentiel des médecins dans les différents CHU. Elle pourrait servir de support pour encourager les médecins à développer la régulation la plus adaptative afin d'être plus efficace dans leur prise en charge du patient. Elle pourrait également contribuer à valoriser le médecin accusé à tors ou à raison par l'opinion publique en mettant en lumière ses efforts quotidiens dans sa pratique afin de sauver des vies. L'objectif est de mieux comprendre l'activité cognitive du médecin ainsi que les interactions qui existent entre les divers processus cognitifs mis en jeu lors de la réalisation d'une tâche. Concrètement, l'étude cherche à comparer deux processus de régulation (proactive et réactive) des médecins sur la base de leur domaine de spécialisation (généraliste ou spécialiste) et de leur ancienneté (débutant ou expérimenté).

L'hypothèse selon laquelle cette étude est conduite stipule que la stratégie de régulation cognitive mise en œuvre par les médecins des CHU varie en fonction de leur domaine de spécialisation et de leur expérience professionnelle. Sur cette base, une méthodologie, des résultats et une discussion sont proposés.

\section{Methodologie}

Pour cette étude, nous nous sommes intéressés au secteur sanitaire public pour deux raisons principales. La première, c'est que la majorité de la population est modeste donc plus à même de fréquenter massivement les centres de santé publics. Cela va amener le médecin à faire des efforts cognitifs pour satisfaire tout ce monde dans des délais raisonnable. La seconde c'est que 
les incidents en rapport avec la conduite des médecins survenus ces dernières années se sont produit dans des CHU et hôpitaux Généraux. Le système sanitaire ivoirien étant à trois niveaux, il serait prétentieux de dire que cette étude va porter sur l'ensemble des médecins du public. Nous portons donc notre attention sur le palier de référence au niveau sanitaire (le troisième niveau) en occurrence les centres hospitaliers universitaires en abrégés CHU.

\section{Cadre d'étude et échantillonnage}

Nous avons la possibilité de trouver des médecins de toutes les spécialités dans les CHU. Ainsi, suite aux procédures administratives, trois des quatre (4) Centres Hospitaliers Universitaires en service en Côte-d'Ivoire ont répondu à notre demande et ont donné leur accord pour que notre étude s'y déroule. Notre population est alors essentiellement constituée de tous les médecins des CHU de Bouaké, de Yopougon et de Treichville. La taille de la population étant considérable, il est nécessaire de faire une sélection dans cette population qui servira de référence.

En effet, l'échantillonnage est une étape extrêmement importante dans une étude scientifique (Guéguen 1997). Vu l'ampleur, les responsabilités et occupations de la population d'étude, et pour des questions de faisabilité, chaque direction médicale et scientifique nous a demandé de faire un choix maximum de trois (03) services pour lesquels ils pourront nous délivrer des attestations pour notre enquête. Nous trouvant ainsi dans l'impossibilité d'interroger tous les médecins de ces trois $\mathrm{CHU}$, le choix des spécialités pour constituer l'échantillon a pris en compte les réalités du milieu. C'est-à-dire, cette restriction des responsables des DMS (Directions Médicales et Scientifiques) limite déjà notre domaine d'action et conditionne notre échantillonnage.

Notre objectif étant de comparer deux modes de régulation, il était primordial d'avoir dans l'échantillon des généralistes de même que des spécialistes. En outre, les spécialités qui traitent des pathologies des adultes diffèrent de celles qui s'intéressent aux enfants. En effet, les patients adultes ont la latitude d'expliquer leur ressenti au médecin, de décrire leur souffrance dans le moindre détail, ce qui n'est pas le cas des enfants. Ces deux catégories de spécialiste ne disposent donc pas des mêmes sources d'information, ce qui les conduira à faire des interprétations différentes en définissant leur mode de régulation préférentiel. Par ailleurs, en portant notre attention sur les estimations faites par l'Organisation Mondiale de la Santé en 2008 sur les maladies chroniques, on remarque que $41 \%$ des adultes âgés de plus de 25 ans souffraient d'hypertension artérielle, $21 \%$ d'hypercholestérolémie et 9\% d'hyperglycémie (OMS, 2011). Ces maladies sont dites du métabolisme car, affectant le système métabolique de l'être humain. Aussi, selon un rapport de l'Unicef, le taux de mortalité infantile en Côte-d'Ivoire est de 108 décès pour 
1000 naissances, soit 12 décès chaque heure (Unicef, 2016). De même, des chercheurs affirment que les principales maladies responsables de décès chez les enfants sont le paludisme (25\%), les infections respiratoires aigües (15\%), les maladies diarrhéiques (9\%) et le VIH/SIDA (3\%), (Liu, Li, Hope, Cousens, Perin, \& al.2012).

Aussi, le plan national sanitaire 2012-2015 révèle que la majorité des motifs de consultation dans les centres de santé concernent les pathologies comme le paludisme, la tuberculose, les maladies du métabolisme telle que le diabète dont la prévalence est à 5,7\%, les affections infantiles et la malnutrition. Les maladies du métabolisme et les pathologies infantiles sont alors en tête de liste en termes de problème de santé.

Au regard de ces statistiques, nous optons pour la méthode empirique pour notre échantillonnage. Elles proviennent selon Guéguen (1997), d'une construction liée à la connaissance préalable des caractéristiques de la population. Ce à quoi correspond notre contexte d'étude en plus du fait qu'elles présentent des avantages de coûts et de rapidité. Selon l'ampleur des services et la présence d'une unité de spécialisation pour les étudiants, nous savons que la référence au niveau des CHU en terme de pédiatrie est le CHU de Treichville et celle en endocrinologie est le CHU de Yopougon.

La pédiatrie et l'endocrinologie sont alors les deux spécialités qui s'imposent à nous dans la réalisation de notre objectif. Nous pourrons alors voir la différence entre ces deux types de spécialistes à travers une comparaison intra-groupe. A ces derniers, l'on ajoute les services de consultation générale afin de faire une comparaison intergroupe (généraliste contre spécialiste). Aussi, en ce qui concerne le CHU de Bouaké, nous avons demandé à travailler avec les pédiatres et les généralistes afin d'avoir un nombre considérable de médecin évoluant dans les mêmes domaines de spécialité. En effet, il est à préciser qu'il n'y a pas d'unité d'endocrinologie au CHU de Bouaké. Notre échantillon est donc composé des médecins pédiatres et généralistes des CHU de Treichville et de Bouaké, ainsi que des médecins endocrinologues et généralistes du CHU de Yopougon ayant acceptés de prendre part à l'étude.

Par ailleurs, nous avons voulu travailler avec des médecins ayant fait le même parcours dans les mêmes conditions académiques. C'est-à-dire considérer les médecins ayant fait leur étude en Côte-d'Ivoire, ce qui les rend équivalent en terme de contenu de la formation reçue durant leur apprentissage de la médecine. Nous avons donc comme échantillon pour cette étude quarante trois (43) médecins dont vingt-trois (23) généralistes et vingt (20) spécialistes (12 pédiatres et 08 endocrinologues). Les médecins interrogés ont des âges compris entre 29 et 62 ans.

Les médecins sont comparés entre eux en fonction des stratégies de régulation mises en œuvre lorsqu'ils sont confrontés à une activité dans 
l'exercice de leur profession. Il faut préciser que l'action nécessite le développement et l'utilisation de diverses stratégies afin de la réussir. Cependant, les individus développent certaines stratégies de façon préférentielle en tenant compte de la situation qui s'annonce. C'est cette stratégie développée qui va orienter le déroulement de l'action et termes de mécanismes qui nécessite sa mise en œuvre. Nous voulons donc voir si l'utilisation d'une stratégie au détriment d'une autre dépend de la spécialité du médecin et de ses années d'expérience. Pour ce faire, nous avons élaboré des instruments qui serviront au recueil des informations nécessaires dans le cadre de cette étude.

\section{Instruments de l'étude}

Nous avons construit un questionnaire que nous avons soumis aux médecins qui constituent notre échantillon d'étude. Ce questionnaire de recueil d'informations qui comprend l'indentification de la spécialisation et de l'expérience est complété par un entretien qui nous sera d'une grande utilité lors de l'interprétation des résultats. Il nous renseigne sur l'âge, la situation matrimoniale, le service, la spécialité, l'année de présence dans le service et les différentes évolutions qui sont intervenues dans la carrière. Ainsi, il nous est possible de repartir les sujets en fonction de leur spécialité, mais aussi de leur expérience professionnelle. L'échelle de régulation cognitive que nous avons élaborée pour évaluer la stratégie de régulation cognitive du médecin accompagne ce questionnaire. Il est présenté simultanément que le questionnaire au médecin et est renseigné avant les questions du guide d'entretien.

Dans notre perspective de recherche, la tâche de prescription de traitement entreprise par le médecin est une situation problème qui nécessite une régulation. En effet, le médecin reçoit beaucoup ou peu d'informations sur le cas qui lui est présenté et doit analyser au mieux celles-ci avant de décider du traitement à proposer après avoir diagnostiqué la pathologie. Cette régulation implique donc la mise en œuvre par ce dernier de conduites de planification, d'organisation et d'anticipation. Ces conduites devraient permettre, à rendre compte des stratégies cognitives de proactivités et réactivités orientées vers le but de la pratique médicale qui est de soulager et soigner le malade qui arrive. L'échelle de régulation cognitive est donc construite et après son analyse factorielle exploratoire et confirmatoire, elle est présentée aux enquêtés. Les répondants étaient donc invités à identifier grâce à cette échelle, la stratégie de régulation cognitive qu'ils utilisent de façon générale dans leur pratique quotidienne. Cette échelle de 12 énoncés est évaluée par une échelle de type Likert en 5 points allant de «presque jamais » à « presque toujours ». La cotation est partie d'un (1) point pour " presque jamais » à cinq (5) points pour «presque toujours » à l'exception des items 
quatre (4) et neuf(9) qui sont cotés à l'inverse. Ces deux questions sont cotées ainsi du fait qu'elles sont les répliques de deux autres questions présentées à l'envers. Il ya cinq (5) questions qui évaluent la proactivité et cinq (5) pour la réactivité. Les items 6 et 12 sont en rapport avec le relationnel. Elles renseignent sur la place et l'importance du soutien sociale dans la corporation des médecins. Elles ont été introduites dans l'échelle car des études ont montré que le soutien social était corrélé positivement avec les stratégies actives et l'efficacité (Rascle 2000). Les individus seront alors considérés comme proactifs ou réactifs selon qu'ils obtiennent un score élevé ou faible à l'un des sous-échelles. La stratégie de régulation mise en œuvre est donc définie dans cette étude par un score.

\section{Resultats}

A la suite des investigations sur le terrain, nous avons obtenu des résultats que nous présentons ici selon l'ordre des trois (03) hypothèses opérationnelles qui ont découlé de notre hypothèse générale.

\section{Domaine de spécialisation et stratégie de régulation}

Tableau 1: Répartition des médecins selon la stratégie de régulation utilisée en fonction du domaine de spécialisation.

\begin{tabular}{lccc} 
& \multicolumn{2}{c}{ Stratégie de régulation cognitive } & \\
Domaine de spécialisation & Réactive & proactive & Total \\
\hline Médecine Générale & 12 & 11 & 23 \\
\hline Spécialité Médicale & 06 & 14 & 20 \\
\hline Total & 18 & 25 & 43 \\
\hline
\end{tabular}

L'analyse du tableau permet de constater que sur 43 médecins interrogés dont 25 sont proactifs, il y en a 14 de spécialité médicale et 11 de médecine générale. Parmi les médecins réactifs au nombre de 18, il y en a 06 de spécialité médicale et 12 de médecine générale. Les effectifs que nous révèlent les différentes cases de ce tableau nous révèlent une différence entre les généralistes et les spécialistes dans la stratégie de régulation développée.

Pour l'analyse statistique, nous soumettons cette relation au test de khi deux (khi 2, khi carré ou encore $\mathrm{X}^{2}$ ). Ce test indique que le Khi deux calculé $\left(\mathrm{X}^{2} \mathrm{cal}=\mathbf{2 , 1 5}\right)$ est inférieur au Khi deux théorique $\left(\mathrm{X}^{2} \mathrm{th}=\mathbf{3 , 8 4}\right)$ lu dans la table des valeurs critiques à 1 degré de liberté et au seuil de probabilité .05 . Il n'y a pas de grande différence entre les effectifs des deux groupes de médecin. La différence entre le khi deux théorique et le khi deux calculé est faible pour attester d'une différence entre le groupe de médecine générale et celui de spécialité médicale. Par conséquent, il n'existe pas de relation entre la stratégie de régulation cognitive des médecins et leur domaine de spécialisation. 


\section{Expérience professionnelle et stratégie de régulation}

Tableau 2: Répartition des médecins selon la stratégie de régulation utilisée en fonction de l'expérience professionnelle

Stratégie de régulation cognitive

\begin{tabular}{lccc} 
Domaine de spécialisation & Réactive & Proactive & Total \\
\hline $\begin{array}{l}\text { Débutants (moins de 05 } \\
\text { ans de présence) }\end{array}$ & 14 & 03 & 17 \\
\hline $\begin{array}{l}\text { Expérimentés (plus de 05 } \\
\text { ans de présence) }\end{array}$ & 04 & 22 & 26 \\
\hline Total & 18 & 25 & 43 \\
\hline
\end{tabular}

Le tableau révèle que l'effectif des médecins de moins de 05 ans de présence dans le service qui sont réactifs (14) est supérieur à celui des médecins de moins de 05 ans de présence dans le service qui sont proactifs(03). En revanche, l'effectif des médecins de plus de 05ans de présence dans le service qui sont réactifs est inférieur (04) à celui de leurs homologues proactifs (22). Les effectifs des différentes cases de ce tableau nous donnent de constater une différence entre les débutants et les expérimentés dans la mise en place de la stratégie de régulation cognitive.

L'analyse statistique indique que le Khi 2 calculé $\left(X^{2}\right.$ cal=18,92) est supérieur au Khi 2 théorique $\left(X^{2}\right.$ th $\left.=\mathbf{3 , 8 4}\right)$ lu dans la table des valeurs critiques à 1 degré de liberté et au seuil de probabilité .05. Il y a une différence significative entre les effectifs des deux groupes de sujets. La comparaison du khi 2 calculé le khi 2 théorique témoigne de l'existence de différence entre les expérimentés et les débutants. Il existe par conséquent, une différence entre les médecins dans la mise en place de stratégie de régulation cognitive en fonction de leur expérience professionnelle.

Domaine de spécialisation, expérience professionnelle et stratégie de régulation 
Tableau 3: Répartition de la stratégie de régulation en fonction du domaine de spécialisation et de l'expérience professionnelle

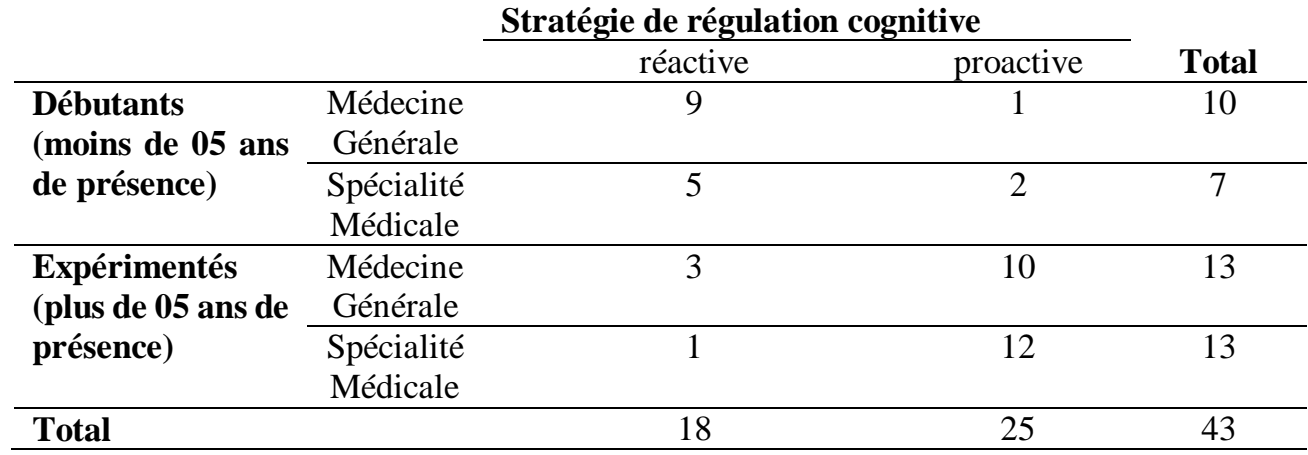

Le nombre des médecins spécialistes expérimenté qui développe une stratégie de régulation proactive (12) est supérieur à celui des généralistes débutants qui développent une stratégie de régulation réactive (9). Celui des généralistes expérimentés qui développe une régulation réactive (3) est supérieur à celui des spécialistes débutants qui développe une régulation proactive (2). Aussi, l'effectif des généralistes expérimentés qui développent une stratégie de régulation proactive (10) est supérieur à celui des spécialistes débutants qui développent une régulation réactive (5). En ce qui concerne le groupe des spécialistes expérimentés qui développent une stratégie de régulation réactive (01), il compte un seul médecin tout comme le groupe des généralistes débutants qui développent une stratégie de régulation proactive (1).

Au niveau statistique, le Khi 2 calculé $\left(X^{2}\right.$ cal=15,17) est supérieur au Khi 2 théorique $\left(\mathrm{X}^{2}\right.$ th $\left.=\mathbf{7 , 8 1}\right)$ lu dans la table des valeurs critiques à 3 degré de liberté et au seuil de probabilité .05. Il y a une différence significative entre les effectifs de ces différents groupes de médecin. Par conséquent, il existe une relation entre la stratégie de régulation cognitive des médecins et l'effet combiné du domaine de spécialisation et de l'expérience professionnelle.

Afin de déterminer la force du lien de dépendance entre la variable stratégie de régulation cognitive et l'effet combiné du domaine de spécialisation et de l'expérience professionnelle, nous avons mesuré l'intensité de ce lien. Pour se faire, nous utilisons le coefficient de Cramer noté $\mathrm{V}$ pour évaluer cette force.

Les règles d'interprétation du $\mathrm{V}$ sont contenues dans le tableau que voici: 


\begin{tabular}{|c|c|}
\hline Valeur du coefficient de Cramer $\mathrm{V}$ & Intensité du lien entre les deux variables \\
\hline $0 \leq \mathrm{V} \leq 0.1$ & Très faible, voire absence de dépendance \\
\hline $0.1 \leq \mathrm{V} \leq 0.3$ & Dépendance modéré \\
\hline $0.3 \leq \mathrm{V} \leq 0.5$ & Dépendance assez forte \\
\hline $0.5 \leq \mathrm{V} \leq 1$ & Dépendance forte \\
\hline
\end{tabular}

Tableau t'interprétation de $\mathrm{V}$

Après calcul, le $\mathrm{V}$ est égal à 0.4 , il appartient à l'intervalle $0.3 \leq \mathrm{V} \leq$ 0.5 . Cela nous permet d'affirmer que l'intensité (force de dépendance) du lien entre l'effet combiné du domaine de spécialisation avec l'expérience professionnelle et la stratégie de régulation cognitive est assez forte.

Pris isolement, la spécialisation n'influence pas la stratégie de régulation. On peut donc déduire que des deux variables, seule l'expérience professionnelle manifeste une force de dépendance assez élevée. Nous pouvons alors conclure que c'est cette force de dépendance qui est à la base de l'intensité du lien entre l'effet combiné des deux variables indépendante et de la variable dépendante.

\section{Discussion des Resultats}

Nous avons évalué la stratégie de régulation des médecins en fonction de leur spécialité et de leur expérience dans la pratique. Les résultats obtenus indiquent dans l'ensemble qu'il y a un rapport entre les variables à l'étude. Pour ce qui est de la relation entre le domaine de spécialisation et la stratégie de régulation, il apparait que dans l'accomplissement de leur tâche, les spécialistes et les généralistes évitent tous la complication de la maladie. Ils répondent aux besoins du patient en temps réel à travers une régulation situationnelle. En ce qui concerne la relation entre l'expérience et la stratégie de régulation, nos résultats laissent conclure que les médecins qui accumulent une présence de plus de cinq (05) ans dans le même service mettent en œuvre une stratégie proactive alors que ceux qui ont moins de cinq (05) ans de présence développent une stratégie réactive. Enfin, les résultats de l'action combinée du domaine de spécialisation et de l'expérience professionnelle attestent que ces deux variables prisent ensemble influencent la tendance des médecins à mettre en œuvre une stratégie de régulation de façon préférentielle. L'on observe donc des médecins spécialistes expérimentés utilisant la stratégie de régulation proactive pour venir à bout de leurs responsabilités, tandis que des médecins généralistes débutants utilisent la stratégie de régulation réactive.

Ces résultats nous révèlent que la stratégie de régulation est un processus mental qui guide l'orientation de toute action. Elle est influencée par plusieurs facteurs, cependant, l'expérience professionnelle est la plus 
importante de tous. En effet, un médecin manifeste une intention avant d'agir, ce qui se concrétise en action lorsque le médecin dispose de suffisamment de connaissances sur la maladie et qu'il ya des conditions dans son environnement favorables à la mise en œuvre de cette action. Cela s'explique par le fait que l'expérience enrichit et procure suffisamment d'assurance pour susciter une action rapide. Elle donne la facilité au médecin de faire le bilan de ses connaissances. Grace aux réussites et aux échecs passés, le praticien a la possibilité de faire des comparaisons selon la pathologie suspectée.

Ces résultats concordent avec ceux de certains chercheurs dans le domaine de la psychologie cognitive. En effet, N'Guessan (1991) dans son travail avec des adolescents Suisse, a fait remarquer que, les stratégies observées chez ces derniers lors d'une activité de résolution de problème sont évolutives. Ses résultats affirment qu'au début de l'activité, les sujets essaient le tout pour réussir sans avoir au préalable quelle démarche sera bénéfique. Cependant, plus ils durent dans la reproduction de cette activité, ils se sentent plus en confiance et préétablissent au fur et à mesure des démarches bien élaborées d'anticipation et de planification. De même, les travaux de Wendpouiré (2016) et ceux de Soule (2014) sur les médecins laissent paraitre que l'expérience du médecin a une influence sur sa productivité. Cette influence est due au fait que l'expérience met à disposition le capital acquis lors des années de pratique antérieur. Concrètement, les résultats de Wendpouiré (op. cit) révèlent que l'expérience affecte la performance des médecins québécois en les rendant plus rapides. Celle de soule (op. cit) indiquent que l'expérience croit sans grand apport sur la productivité pendant les cinq premières années environ et ne dévient bénéfique qu'à cet instant où le médecin commence à s'en servir en se référant aux résultats antérieurs.

Sachant que la productivité prend en compte l'efficacité et la rapidité (source d'anticipation), le médecin le plus productif est alors celui qui fait preuve de proactivité. Ces résultats confirment donc nos hypothèses numéro 2 et numéro 3. Les médecins expérimentés de nos travaux sont efficaces et rapides dans leurs démarches et procédures. Ces médecins utilisent les habilités issus de leurs pratiques passées en tant que débutants pour améliorer leur productivité à travers des stratégies de régulations proactives. Les résultats de l'Hypothèse un convergent quant à elles vers la recherche de Ayirir (2011) sur la fréquence d'utilisation des stratégies selon le niveau de compétence des apprenants. Les apprenants dans l'étude de Ayirir (op. cit) tout comme les médecins dans la notre disposent de diverses stratégies et les utilisent toutes mais en alternance selon des conditions facilitantes et non selon la spécialisation.

Ainsi, peu importe leur domaine de spécialisation, les médecins tiennent compte de leur environnement professionnel et des possibilités d'action que leur offre cet environnement. Par conséquent, la stratégie de 
régulation mise en œuvre est fonction de l'état de santé du patient (urgence ou pas), mais aussi, des ressources environnementales susceptibles de faciliter ou non l'action thérapeutique. Néanmoins, plus les médecins sont habitués à leur environnement professionnel, plus ils se servent des réussites et échecs des actions passées pour mieux appréhender les cas qui se présentent à eux. L'expérience professionnelle est donc essentielle dans le processus de régulation, plus particulièrement dans le contexte de l'accomplissement d'une tâche spécifique.

\section{Conclusion}

Cette étude nous a permis d'apprécier la force d'influence de l'expérience professionnelle sur la stratégie de régulation cognitive. Cette expérience donne l'assurance au médecin et la capacité d'agir de façon efficace en mettant en place une stratégie de régulation proactive. Bien que la stratégie de régulation réactive soit moins valorisée, elle peut néanmoins se trouver efficace et pertinente selon la situation. Le désir premier du médecin, c'est de trouver un traitement qui vient à bout de la pathologie qui lui est soumis. Cependant, les conditions de travail de ces derniers dans les Centres Hospitaliers Universitaires et l'état de santé des patients qui arrivent ne sont pas faites pour faciliter la planification et les anticipations. De ce fait, c'est l'expérience qui procure au médecin un cadre propice d'analyse pertinent. Grace aux réussites passées, le médecin a confiance en ses capacités et en l'efficacité de ses choix thérapeutiques. Ce qui le conduit à un traitement optimal des informations à disposition et à une réduction de la charge cognitive permettant ainsi l'adoption de la stratégie qui convient le mieux.

\section{References:}

1. Adeyoyin, Samuel Olu; Agbeze-Unazi, Florence; Oyewunmi, Olatundun Oluwatoyin; Adegun, Adewale Isau; and Ayodele, Rafiu Olabamiji, (2015). Effects of Job Specialization and Departmentalization on Job Satisfaction among the Staff of a Nigerian University Library. Library Philosophy and Practice (e-journal). 1295.

2. Ayirir, İ. O. (2011). La cognition et les stratégies cognitives dans l'apprentissage des langues étrangères. Hacettepe Üniversitesi Ĕgitim Fakültesi Dergisi, 40(40).

3. Bini, Konan Diby, Kouassi-Gohou (2008). Rapport d'évaluation $d u$ système national d'information sanitaire ivoirien par l'outil du réseau de métrologie sanitaire RMS/HMN. Ministère de la santé et de l'hygiène publique 
4. Boekaerts, M. (1997). Self-Regulated Learning : a new concept embraced by researchers, policy makers, educators; teachers and students. In Learning and Instruction, 7(2), 161-186.

5. Boss, G. (2000). La fin de l'ordre économique. Editions du Grand Midi.

6. Brick, N., MacIntyre, T., and Campbell, M. (2015). Metacognitive processes in the self-regulation of performance in elite endurance runners. Psychology Sport Exercice. 19, 1-9.

7. Buchs, C., Darnon, C., Quiamzade, A., Mugny, G., \& Butera, F. (2008). Conflits et apprentissage. Régulation des conflits sociocognitifs et apprentissage. Revue Française de Pédagogie, 163, 105-125.

8. Chalus-Sauvannet, M. C. (2000). Dynamisation du dispositif de veille stratégique pour la conduite de stratégies proactives dans les entreprises industrielles (Doctoral dissertation, Lyon 2).

9. Chiswick, B. R. (2003). Jacob Mincer, experience and the distribution of earnings. Review of Economics of the Household, 1(4), 343-361.

10. Duggan, G. B., \& Payne, S. J. (2008, April). Knowledge in the head and on the web: Using topic expertise to aid search. In Proceedings of the SIGCHI conference on Human factors in computing systems (3948). ACM.

11. Efklides, A. (2006). Metacognitive experiences: The missing link in the self-regulated learning process. Educational Psychology Review, 18(3), 287-291.

12. Flavell, J. H. (1976). Metacognitive aspects of problem solving. The nature of intelligence, 12, 231-235

13. Gombert, J. É. (1990). Le développement métalinguistique. Psychologie d'aujourd'ui.

14. Guéguen, N. (1997). Manuel de statistique pour psychologues. Paris : Dunod.

15. Houdé O., Kayser D., Koenig O., Proust J., Rastier F. \& coll (2003). Vocabulaire de sciences cognitives. Quadrige Puf

16. Hurley, J., \& Labelle, R. (1995). Relative fees and the utilization of physicians' services in Canada. Health Economics, 4(6), 419-438.

17. Koriat, A., \& Levy-Sadot, R. (2000). Conscious and unconscious metacognition: A rejoinder.

18. Lemaire, P., \& Reder, L. (1999). What affects strategy selection in arithmetic? The example of parity and five effects on product verification. Memory \& Cognition, 27(2), 364-382.

19. Lemelin, C. (1998). Économie de l'éducation. Presses Universitaires du Québec. 
20. Liu, Li, Hope L. Johnson, Simon Cousens, Jamie Perin, Susana Scott, Joy E. Lawn, Igor Rudan, Harry Campbell, Richard Cibulskis, et Mengying Li. (2012). "Global, Regional, and National Causes of Child Mortality: An Updated Systematic Analysis for 2010 withTime Trends since 2000." The Lancet 379 (9832): 2151-2161

21. Locke, E., \& Latham, G. (2002). Building a practically useful theory of goal setting and task motivation: a 35-year odyssey. American Psychologist, 57(9), 705-717.

22. Locke, E., \& Latham, G. (2006). New directions in goal-setting theory. Current Directions in Psychological Science, 15(5), 265-268.

23. Mahé De Boislandelle, H. (1998). GRH en PME: universalité et contingences: essai de théorisation. Revue internationale PME: Économie et gestion de la petite et moyenne entreprise, 11(2-3), 1130.

24. Ministère de la Santé et de la Lutte contre le SIDA, (2012). Plan National de Développement 2012-2015. Abidjan, République de Côte d'Ivoire.

25. Morin, E. (2000). Les sept savoirs nécessaires à l'éducation du futur. Paris: Seuil.

26. N'guessan A. G. (1991). L'apprentissage d'un jeu de stratégies typiquement africain (L'awèlé) chez les adolescents et les joueurs d'échecs Suisses. Thèse de Doctorat de Psychologie, non publiée, Faculté des Lettres. Fribourg : Université de Fribourg.

27. Noël, B., Romainville, M., Wolfs, J. L., \& Wolf, J. L. (1995). La métacognition: facettes et pertinence du concept en éducation. Revue française de pédagogie, 47-56.

28. OLIVE, L. (2011). Stress professionnel et stratégies d'ajustement de l'interprète français-langue des signes.

29. Rascle, N. (2000). Testing the mediating role of appraisal stress and coping strategies on employee adjustment in a context of job mobility. European Review of Applied Psychology, 50, 3, 301-307.

30. Richard, J. F. (1990). Les activités mentales. Comprendre, raisonner, trouver des solutions. Paris: Armand Colin.

31. Royer, J. M., Cisero, C. A., \& Carlo, M. S. (1993). Techniques and procedures for assessing cognitive skills. Review of Educational Research, 63(2), 201-243.

32. Samurçay, R., \& Rabardel, P. (2004). Modèles pour l'analyse de l'activité et des compétences, propositions. Recherches en didactique professionnelle, 163-180.

33. Schneider, W., \& Lockl, K. (2002). 10 The development of metacognitive knowledge in children and adolescents. Applied metacognition, 224. 
34. Son, L. K., \& Schwartz, B. L. (2002). The relation between metacognitive monitoring and control. Applied metacognition, 15-38.

35. Soule, I. (2014). Impact de l'expérience professionnelle sur la productivité des médecins Omnipraticiens au Québec. Mémoire de maîtrise en économie. Université Laval, Québec.

36. Swinnen, S. P., Massion, J., Heuer, H., \& Casaer, P. (Eds.). (2013). Interlimb coordination: neural, dynamical, and cognitive constraints. Academic Press.

37. Wendpouiré, Adele B. (2016). Impact de l'expérience professionnelle sur la productivité des médecins specialists au Québec. Mémoire de maîtrise. Université Laval.

38. Zimmerman BJ (1989). A social cognitive view of self-regulated academic learning. Journal of Educational Psychology 91:329-339 https://www.goafricaonline.com/ci/articles/27-administration-santecote-ivoire 